\title{
Integration of Lobula Plate Output Signals by DNOVS1, an Identified Premotor Descending Neuron
}

\author{
Juergen Haag, Adrian Wertz, and Alexander Borst \\ Department of Systems and Computational Neurobiology, Max-Planck-Institute of Neurobiology, D-82152 Martinsried, Germany
}

\begin{abstract}
Many motion-sensitive tangential cells of the lobula plate in blowflies are well described with respect to their visual response properties and the connectivity among them. They have large and complex receptive fields with different preferred directions in different parts of their receptive fields matching the optic flow that occurs during various flight maneuvers. However, much less is known about how tangential cells connect to postsynaptic neurons descending to the motor circuits in the thoracic ganglion and how optic flow is represented in these downstream neurons. Here we describe the physiology and the connectivity of a prominent descending neuron called DNOVS1 (for descending neurons of the ocellar and vertical system). We find that DNOVS1 is electrically coupled to a subset of vertical system cells. The specific wiring leads to a preference of DNOVS1 for rotational flow fields around a particular body axis. In addition, DNOVS1 receives input from interneurons connected to the ocelli.
\end{abstract}

Key words: motion detection; insect; vision; descending; gap junction; flow field

\section{Introduction}

For visual course control, flies rely on a set of motion-sensitive neurons called lobula plate tangential cells (LPTCs). LPTCs represent $\sim 60$ large neurons per brain hemisphere, each of which can be identified individually because of its invariant anatomy and characteristic visual response properties (Pierantoni, 1976; Hausen, 1977; Hausen, 1982; Hengstenberg et al., 1982; Borst and Haag, 1996). Among them, cells are found responding preferentially to vertical motion, such as vertical system (VS) cells, as well as cells that are best activated by horizontal motion, such as the horizontal system (HS) cells. Per hemisphere, there exist three HS cells (the northern HSN, the equatorial HSE, and the southern HSS cell) and $10 \mathrm{VS}$ cells (VS1-VS10), together covering almost completely the visual space surrounding the animal. LPTCs often have complex receptive fields, with different preferred direction in different parts of the visual field matching the optic flow that occurs during specific flight maneuvers of the fly (Krapp and Hengstenberg, 1996; Krapp et al., 1998; Franz and Krapp, 2000; Karmeier et al., 2003). HS and VS cells are the major output elements of the lobula plate. They convey flow-field information onto descending neurons that ultimately control motor neurons for locomotion or head movements.

Over the past decades, LPTCs have been studied extensively with respect to their visual response properties (Hausen, 1977,

Received 0ct. 9, 2006; revised Dec. 15, 2006; accepted Dec. 16, 2006.

This work was supported by the Max-Planck-Society, a grant from the Bundesministerium für Bildung und Forschung to the Bernstein Center Munich (J.H.), and Deutsche Forschungsgemeinschaft Grant GRK 1091 (A.W.). We are grateful to Renate Gleich, Marianne Braun, and Dietmute Bueringer for excellent technical assistance.

Correspondence should be addressed to Juergen Haag, Department of Systems and Computational Neurobiology, Max-Planck-Institute of Neurobiology, Am Klopferspitz 18, D-82152 Martinsried, Germany. E-mail: haag@neuro.mpg.de.

DOI:10.1523/JNEUROSCI.4393-06.2007

Copyright $\odot 2007$ Society for Neuroscience $\quad$ 0270-6474/07/271992-09\$15.00/0
1981; Hengstenberg, 1977; Hengstenberg et al., 1982; Eckert and Dvorak, 1983; Haag, 1994; Haag et al., 1999) (for review, see Hausen, 1984; Borst and Haag, 2002) and the connectivity among them (Hausen, 1981, 1984; Horstmann et al., 2000; Haag and Borst, 2001, 2002, 2003, 2004, 2005; Farrow et al., $2003,2005,2006)$. However, less is known about the neurons to which LPTCs project. These descending neurons have been mainly described anatomically (Strausfeld and Bassemir, 1985a,b; Strausfeld and Seyan, 1985; Milde and Strausfeld, 1986; Strausfeld et al., 1987), but little is known about their physiology (but see Gronenberg and Strausfeld, 1990; Strausfeld and Gronenberg, 1990; Gilbert et al., 1995; Gronenberg et al., 1995; Chan et al., 1998; Huston and Krapp, 2003) and their specific connectivity to LPTCs.

The anatomy of descending neurons has been investigated mainly by cobalt backfills in which either the cervical connective was bathed in a cobalt solution or the cobalt solution was iontophoretically injected into the cervical connective. This led, besides many other descending neurons, to the staining of a prominent group of three Y-shaped cells termed DNOVS (for descending neurons of the ocellar and vertical system) (Strausfeld and Bassemir, 1985a). Ocelli are light-sensitive organs on the dorsal surface of the head. The three ocelli form a triangle, and each one has a single wide-angle lens. With the photoreceptor array being out of focus, they appear to be suited for detecting changes in overall brightness (Schuppe and Hengstenberg, 1993). The DNOVS project with their axons through the cervical connective into the prothoracic ganglion. The cobalt fill revealed dye coupling between a subset of VS cells (VS4-VS9) and DNOVS1. In their study, Strausfeld and Bassemir (1985a) found that most of the contacts between VS cells and DNOVS1 are mediated by chemical synapses. In addition, however, gap-junction-like appositions were found in close vicinity to chemical synapses. Besides the input from VS cells, additional input to DNOVS1 from 
ocellar interneurons, so called L-neurons (Simmons et al., 1994), was suggested.

In this article, we present electrophysiological data on a specific descending neuron, the DNOVS1 (also called DNDC 1-1 by Gronenberg et al., 1995). Given the described connectivity to VS cells, we studied how the signals of a subset of VS cells and ocellar input are integrated on the dendrite of DNOVS1.

\section{Materials and Methods}

Preparation and setup. Female blowflies (Calliphora vicina) were briefly anesthetized with $\mathrm{CO}_{2}$ and mounted ventral side up with wax on a small preparation platform. The head capsule was opened from behind; the trachea and air sacs that cover the lobula plate were removed. To eliminate movements of the brain caused by peristaltic contractions of the esophagus, the proboscis of the animal was cut away and the gut was pulled out. This allowed stable intracellular recordings of up to $45 \mathrm{~min}$. The fly was then mounted on a heavy recording table looking down onto the stimulus monitors. The fly brain was viewed from behind through a fluorescence microscope (Axiotech Vario $100 \mathrm{HD}$; Zeiss, Oberkochen, Germany).

Stimulation. Stimuli were generated on Tektronix (Wilsonville, OR) 608 monitors by an image synthesizer (Picasso, Innisfree) and consisted of a one-dimensional grating of $16.7^{\circ}$ spatial wavelength and $87 \%$ contrast displayed at a frame rate of $200 \mathrm{~Hz}$. The mean luminosity of the screen was $11.2 \mathrm{~cd} / \mathrm{m}^{2}$. The intensity of the pattern was square-wave modulated along its vertical axis. For measuring the sensitivity along the azimuth (see Fig. 1), we used three Tektronix monitors: monitor 1 was placed contralateral and extended from -90 to $-30^{\circ}$ in the horizontal direction and from +40 to $-30^{\circ}$ in the vertical direction, monitor 2 was placed at position -15 to $+40^{\circ}$, and monitor 3 was at position +55 to $+120^{\circ}$. Each monitor screen was divided into five stripes each with a horizontal extent of $11^{\circ}$ for monitor 2 and $13^{\circ}$ for monitor 3 . The pattern inside these stripes could be moved independently. For all other experiments (see Figs. 2, 8), we used two Tektronix monitors. The stimulus field extended from +16 to $+42^{\circ}$ and from +95 to $+133^{\circ}$ along the azimuth and from -30 to $+30^{\circ}$ in elevation of the fly.

For stimulating the ocelli, we used a blue $\left(\lambda=466 \mathrm{~nm}, 72 \mathrm{~cd} / \mathrm{m}^{2}\right)$ light-emitting diode (LED) (LB3336; Osram, Munich, Germany) placed in front of the ocelli at a distance of $2 \mathrm{~mm}$. Shielding the LED ensured that the facet eyes did not become stimulated by the LED. This was also confirmed by experiments in which we occluded the ocelli. Under these conditions, switching on the LED did not elicit a response in DNOVS1.

Electrical recording. For intracellular recordings of the cells, electrodes were pulled on a Brown-Flaming micropipette puller (P-97) using thinwalled glass capillaries with an outer diameter of $1 \mathrm{~mm}$ (GC100TF-10; Clark Electromedical Instruments, Reading, UK). The tip of the electrode was filled with $10 \mathrm{~mm}$ Alexa 488 (Invitrogen, Carlsbad, CA). The shaft of the electrode was filled with a $2 \mathrm{~m}$ KAc solution. Electrodes had resistances of $\sim 15 \mathrm{M} \Omega$. For dual intracellular recordings, one electrode was filled with the green fluorescent dye Alexa 488, and the other electrode was filled with the red fluorescent dye Alexa 568 (Invitrogen). For the Neurobiotin staining, the tip of the electrode was filled with a mixture of 3\% Neurobiotin (Vector Laboratories, Burlingame, CA) and 3\% fluorescein. A SEL10 amplifier (NPI Electronic, Tamm, Germany) operated in the bridge mode was used throughout the experiments. In the experiments with dual intracellular recordings, we used an additional SEL10 amplifier. For data analysis, the output signals of the amplifiers were fed to a Pentium III personal computer via an 12 bit analog-to-digital converter (DAS-1602/12; ComputerBoards, Middleboro, MA) at a sampling rate of $5 \mathrm{kHz}$ and stored to hard disc. The signals were evaluated off-line by a program written in Delphi (Borland, Buffalo, NY).

The response to motion stimuli was calculated by averaging the membrane potential from $200 \mathrm{~ms}$ after the onset of the motion stimulus over $800 \mathrm{~ms}$. The membrane potential averaged over $500 \mathrm{~ms}$ before the onset of the motion stimulus was then subtracted from this value.

Normalized cross-correlograms (see Fig. $5 b, c$ ) were calculated from the first $800 \mathrm{~ms}$ of the membrane potentials according to Kimpo et al.
(2003). Defining the cross-correlation CC $(\tau)$ between two responses $r_{A}$ and $\mathrm{r}_{\mathrm{B}}$ as

$$
C C(\tau)=\left\langle\frac{1}{T} \int_{0}^{T} d t \cdot r_{\mathrm{A}}(t) \cdot r_{\mathrm{B}}(t+\tau)\right\rangle,
$$

and the total power $\sigma_{X}^{2}$ of $r_{X}(t)$ as

$$
\sigma_{X}^{2}=\left\langle\frac{1}{T} \int_{0}^{T} d t \cdot r_{X}^{2}(t)\right\rangle,
$$

the normalized cross-correlation $h(\tau)$ is then

$$
h(\tau)=\frac{C C(\tau)}{\sqrt{\sigma_{A}^{2} \cdot \sigma_{B}^{2}}} .
$$

For recording from DNOVS1, a VS cell was filled with Alexa 488 and visualized under fluorescence light. The VS cell then served as a landmark for finding the DNOVS1 neuron. All of the recordings were made from the dendritic region of DNOVS1 and from the axons of the VS cells. VS cells were identified using a method described by Farrow (2005). There, not only the specific anatomy of the cell was taken into account but also the relative position of their ventral dendrite within the lobula plate.

Two-photon microscopy. For registering the anatomy of DNOVS1, we used a custom-built two-photon microscope (Denk et al., 1990; Haag et al., 2004) consisting of the following components: a 5 W pumped titanium:sapphire laser (MaiTai; Spectra Physics, San Jose, CA), a Pockels cell (Conoptics, Danbury, CT), scan mirrors including drivers (Cambridge Technology, Lexington, MA), a scan lens (4401-302; Rodenstock, Columbus, OH), a tube lens (MXA 22018; Nikon, Tokyo, Japan), a dichroic mirror (DCSPR $25.5 \times 36$; AHF, Tuebingen, Germany), and a $40 \times$ water immersion lens (Zeiss). The lens can move along all three axes by a step-motor-driven micromanipulator (MP285-3Z; Sutter Instruments, Novato, CA). Emitted light is filtered in parallel by two bandpass filters (HQ 535/50M and HQ 610/75M; Chroma Technology, Brattleboro, VT) and collected by multi-alkali photomultipliers (R6357; Hamamatsu, Bridgewater, NJ). The whole system is controlled by custom-written software (CfNT version 1.569; Michael Mueller, Max-Plank-Institute for Medical Research, Heidelberg, Germany). Three-dimensional reconstruction of the cells was performed with the software package AMIRA version 4.0 (Mercury Computer Systems, Berlin, Germany).

Histology. After filling a cell with the Neurobiotin and fluorescein mixture, it was identified under the fluorescence microscope. The fly was then kept at $+4^{\circ} \mathrm{C}$ for at least $60 \mathrm{~min}$ to allow for diffusion of Neurobiotin to coupled cells. The head of the fly was then cut off and fixed for 24-48 $\mathrm{h}$ at $4^{\circ} \mathrm{C}$ in $4 \%$ paraformol and $0.2 \%$ picric acid mixture in $0.15 \mathrm{~m}$ phosphate buffer. The head was washed in PBS buffer, and the brain was taken out. The brain was then frozen in liquid nitrogen for $20 \mathrm{~s}$. After several rinses with PBS, the brain was incubated with Vectastain $\mathrm{ABC}$ kit (Vector Laboratories) overnight. Before incubation in a $0.025 \% \mathrm{CoCl}_{2}$ and $0.02 \%$ $\mathrm{NiCl}_{2}$ mixture in PBS buffer for $30 \mathrm{~min}$, the brain was rinsed several times in PBS buffer. The diaminobenzidine reaction was started by transferring the tissue in a solution containing $0.05 \% \mathrm{CoCl}_{2}, 0.02 \% \mathrm{NiCl}_{2}$, and $0.01 \%$ $\mathrm{H}_{2} \mathrm{O}_{2}$ for $10 \mathrm{~min}$ at room temperature. The brain was then washed again in PBS buffer and dehydrated in alcohol before embedding it in mixture of distyrene, tricresyl phosphate, and xylene. The stained cells were identified under a dissection microscope (MZFLIII; Leica, Nussloch, Germany). Pictures were taken with a CCD camera (DC 320; Leica).

\section{Results}

In a first set of experiments, we measured the responses of DNOVS1 to upward and downward motion at different azimuth positions. Figure $1 a$ shows a schematic drawing of the setup we used. As VS cells, DNOVS1 responds to motion stimuli with a graded shift in membrane potential. As an example, the response to vertical pattern movement at $120^{\circ}$ azimuth is shown (Fig. 1b). Downward motion of the pattern leads to a depolarization, and 
upward motion leads to a hyperpolarization of the cell. Although the responses of DNOVS1 look similar to the responses of VS cells, there are some differences. In contrast to VS cells, no indications for active membrane properties can be found in the response to motion (but see Fig. 7). In none of the recordings could action potentials in response to visual motion stimuli be detected. In addition, without any motion stimuli, there seems to be massive input from presynaptic neurons leading to a rather unstable resting potential. The response to upward and downward motion at different azimuth positions is shown in Figure $1 c$. DNOVS1 did not respond to motion in front of the contralateral eye (position -90 to $-30^{\circ}$ ). The strongest response was found for lateral to caudal stimulus positions. Because of technical limitations, it was not possible to measure the response to monitor positions more caudal than $120^{\circ}$. Besides the response to stimuli in the laterocaudal field, there was a significant response to upward motion in the frontal part of the receptive field. At this frontal position, the response to upward and downward motion was not symmetrical (Fig. 2, columns 3, 4). In general, the responses to visual motion stimuli at the positions tested were rather small compared with the responses found in VS cells.

The measured sensitivity profile with an upward sensitivity in the frontal region and a downward sensitivity in the laterocaudal region points toward a rotation selective flow field. To test this, we recorded the response of DNOVS1 to simultaneous motion stimuli shown at different locations in the receptive field. To do so, we used two stimulus monitors: one placed in front of the fly and a second one in the lateral visual field (Fig. $2 a$ ). Figure $2 b$ shows the response of DNOVS1 to different combinations of motion stimuli. The four left columns show the responses to single stimuli, and the four right columns show responses to combinations of motion stimuli. In agreement with the data shown in Figure $1 c$, DNOVS1 responds with a depolarization to downward motion in the lateral monitor (dark gray, first column) and to upward motion in the frontal monitor (fourth column). The combined motion stimulus (frontal upward and lateral downward) elicited the strongest depolarization (Fig. $2 b$, right column). This indicates that DNOVS1 is tuned more to a rotational flow field than to a translatory one. The comparison between the measured responses to the combined stimuli (right columns) and the arithmetic sum of the responses to single stimuli (light gray columns) indicate a nearly linear summation of the individual responses.

To investigate the connectivity between VS cells and DNOVS1, we performed dual intracellular recordings from different VS cells and DNOVS1, injecting current into one cell and recording the potential response in the other. As an example, Figure $3 a$ shows the membrane potential of DNOVS1 in response to current injection into a VS7 cell. Negative current injection led to a hyperpolarization of DNOVS1, and positive current led to a depolarization of DNOVS1. Thus, current of both polarities is transmitted from VS7 onto DNOVS1. The next experiment demonstrates that the connection works both ways: when the current was injected into DNOVS1, VS7 became depolarized or hyper-
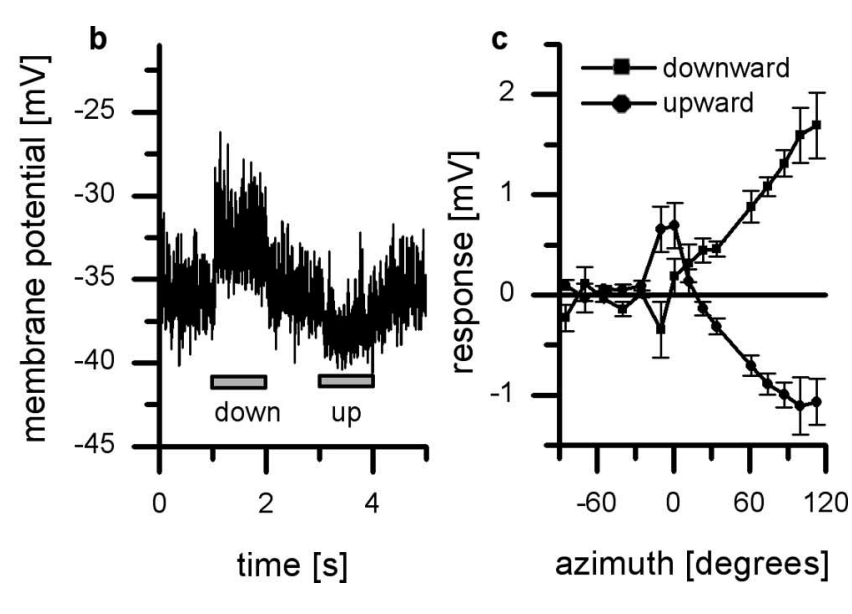

azimuth [degrees]

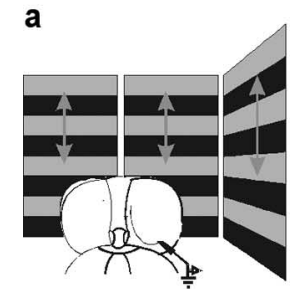

time $[\mathbf{s}]$

Figure 1. Intracellular recording from DNOVS1. $\boldsymbol{a}$, Schematic drawing of the stimulation. $\boldsymbol{b}$, Example response of DNOVS1 to vertical motion at an azimuth position of $120^{\circ}$. The cell responds to downward motion with a depolarization of the membrane potential and to upward motion with a hyperpolarization of the membrane potential. The response is superimposed by massive 政 a

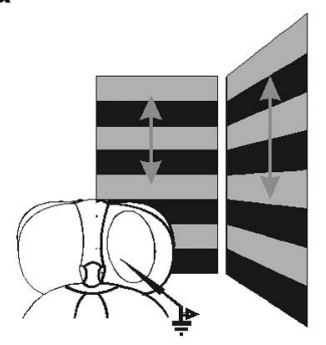

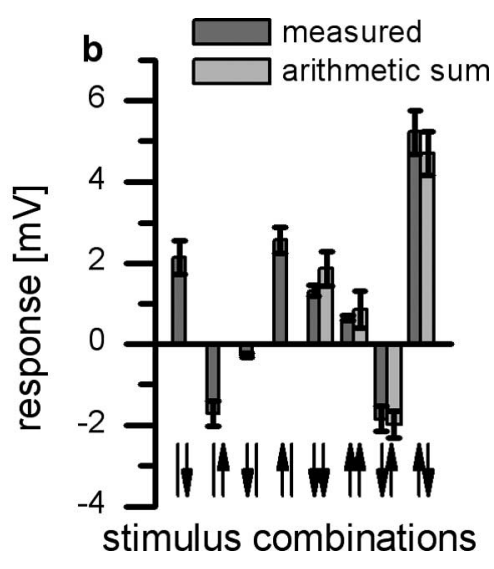

Figure 2. Responses of DNOVS1 to simultaneous motion in two sectors of the receptive field. $\boldsymbol{a}$, Schematic drawing of the stimulus situation. $\boldsymbol{b}$, Averaged responses of DNOVS1 to simultaneous motion stimuli in both monitors (dark gray columns). The neuron responds best to a rotatory motion stimuli consisting of upward motion in the frontal and downward motion in the lateral eye field (rightmost stimulus configuration). Light gray columns show the arithmetic sum of the responses to single stimuli. The measured and the calculated responses to combined stimuli are almost identical. Data represent the mean \pm SEM recorded from $n=4$ flies.

polarized, respectively, depending on the sign of current injection (Fig. $3 b$ ). This bidirectional coupling of the two cells speaks in favor of electrical synapses between them.

In a series of experiments, we investigated the connectivity between all VS cells (except VS10) and DNOVS1 within one brain hemisphere. In general, current injection into VS cells led to a change of the membrane potential of DNOVS1 and vice versa. However, the coupling strength between the different VS cells and DNOVS1 varied considerably. Current injection into VS1VS3 elicited only weak responses in DNOVS1. The strongest coupling could be found between VS6/VS7 and DNOVS1 (Fig. 4).

The similarity between the voltage traces of both cell types when no stimulus was provided also suggested the coupling between VS cells and DNOVS1. Figure $5 a$ shows a double recording of the membrane potentials of DNOVS1 and VS7 under these conditions. We quantified the similarity by calculating the crosscorrelation function between the signals from both cells (Fig. 5b). 
a

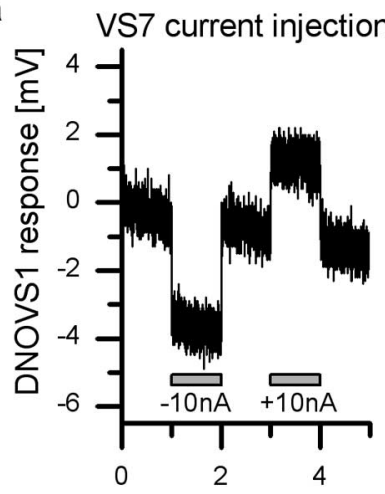

time [s]

\section{b DNOVS1 current injection}

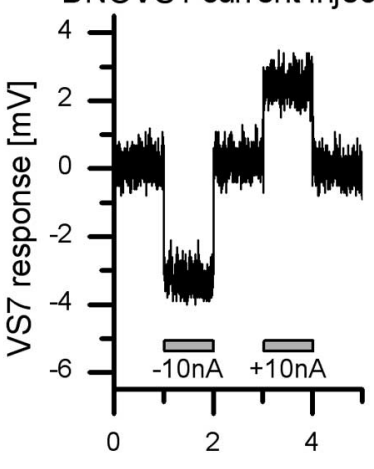

time $[\mathrm{s}]$

Figure 3. Dual intracellular recording from DNOVS1 and VS7. $\boldsymbol{a}$, Current injection of -10 and $+10 \mathrm{nA}$ into VS7 led to a hyperpolarization and depolarization of DNOVS1, respectively. $\boldsymbol{b}$, Same as $\boldsymbol{a}$, but current was injected into DNOVS1.

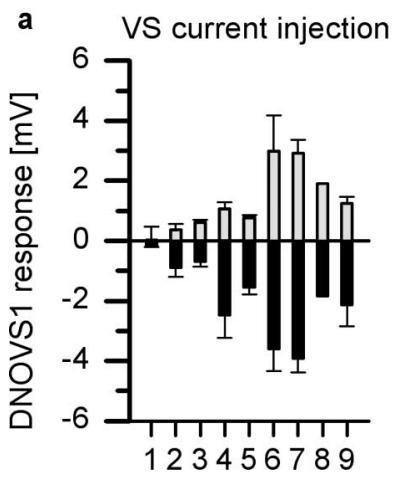

VS-cell number

\section{b DNOVS1 current injection}

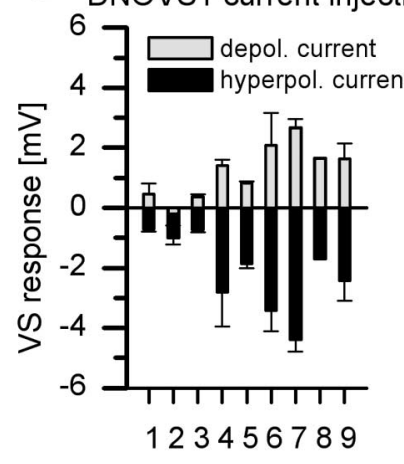

VS-cell number

Figure 4. Double intracellular recording from DNOVS1 and different VS cells. $\boldsymbol{a}$, Current injection of $-10 \mathrm{nA}$ (black bars) and $+10 \mathrm{nA}$ (gray bars) in different VS cells elicited different levels of hyperpolarization and depolarization DNOVS1. Whereas DNOVS1 showed only weak responses to the current injection in VS1-VS3, it responded more strongly when current was injected into VS4 -VS9. The strongest response was found for current injection into VS6 and VS7. $\boldsymbol{b}$, Same as $\boldsymbol{a}$, but current was injected into DNOVS1 and the response was measured in different VS cells. As before, the strongest response could be found in VS6 and VS7. These experiments demonstrate that not only current of both polarities is transmitted between DNOVS1 and VS cells, but that the connection also works in both directions. Data represent the mean $\pm \operatorname{SEM}$ of VS1 $(n=2), \operatorname{VS2}(n=2), \operatorname{VS3}(n=6), \operatorname{VS} 4(n=2), \operatorname{VS5}(n=2), \operatorname{VS6}(n=2)$, VS7 $(n=5)$, VS8 $(n=1)$, and VS9 $(n=3)$.

We found a strong positive correlation between the two signals that peaks at 0 time lag. The peak is almost symmetrical at $\sim 0$ and has a half-width of only $3.2 \mathrm{~ms}$. The plot of the correlation peaks for the different VS cells and DNOVS1 (Fig. $5 c$ ) revealed a pattern similar to the one obtained from current injection experiments (compare with Fig. 4): whereas the membrane potential of VS1VS3 and DNOVS1 showed only a weak correlation, the signals of VS6-VS8 and DNOVS1 turned out to be strongly correlated.

Knowing the connectivity and coupling strength between the various VS cells and DNOVS1, we asked the question how the input from VS cells is integrated on DNOVS1. We therefore measured the response of VS cells to the exact same set of stimuli we used before to characterize DNOVS1 (Fig. 1a). Figure $6 a$ shows the sensitivity of the VS cell responses to downward and $b$ to upward motion along the azimuth in a false color code. Because none of the VS cells showed a response to contralateral azimuth positions $\left(-90\right.$ to $\left.-30^{\circ}\right)$, we did not plot the data for these azi-

muth positions. The peak of the sensitivity to downward and upward motion shifts from frontally to caudally for VS1 to VS9, respectively. In addition, VS7-VS9 showed a sign reversal in their response to motion in the frontal visual field. The measured responses of the VS cells are in agreement with the results of Krapp et al. (1998) who used small dots to map the preferred orientation. The response profile for a given VS cell was then multiplied with the measured coupling strength to DNOVS1 (Fig. 4) and integrated over all VS cells. This gave an estimate of the sensitivity for a neuron that integrates linearly the output of VS cells. The comparison between this estimated sensitivity and the measured sensitivity for vertical pattern motion of DNOVS1 along the azimuth is shown in Figure $6 c$. For stimuli moving downward, the expected sensitivity matches quite well the measured sensitivity of DNOVS1. As for DNOVS1, the responses are almost 0 for frontal stimulus positions and are biggest for laterocaudal stimuli. In contrast, the expected responses for upward motion differ especially for frontal stimuli ( -10 to $+1^{\circ}$ azimuth): whereas DNOVS1 responded with a depolarization to frontal upward motion, the calculated responses were almost 0 at these azimuth positions.

Based on anatomical findings, Strausfeld and Bassemir (1985a) suggested that, in addition to input from VS cells, DNOVS1 also receives input from the ocelli via L-neurons. For measuring the responses to the ocellar input, we used an LED to illuminate the ocelli. Figure $7 a$ shows the response of DNOVS1 to a $500 \mathrm{~ms}$ light pulse emitted by the LED. DNOVS1 responds to the onset of this stimulus with a transient hyperpolarization followed by fast depolarizing deflections of the membrane potential. Switching off the LED leads to a characteristic off response. It consists of a fast depolarization followed by a slow decay back to the resting membrane potential. To test whether the fast depolarizing off response is attributable to the activation of intrinsic voltage-gated currents or simply reflects a passive postsynaptic potential, we hyperpolarized and depolarized the membrane of DNOVS1 by injecting steady-state negative or positive current and measured the off response to the ocellar stimulation under these different conditions. In the case that voltage-dependent currents contribute to the fast depolarization in the off response, strong hyperpolarizing current injection should prevent their activation. Depolarizing current injection should lead to an inactivation of the voltage-dependent currents. If the response is solely attributable to synaptic input without any active amplification, the amplitude of the fast transient should depend linearly on the injected current. Figure $7 b$ shows the result of such an experiment. Plotted is the amplitude of the fast deflection after switching off the light stimulus as a function of the steady current injection. For steady current injections between -10 and $+5 \mathrm{nA}$, the amplitude of the fast depolarization followed almost linearly the amount of injected current. For current injections more negative than $-15 \mathrm{nA}$, however, the amplitude of the peak decreased. This nonlinear dependence of the off response peak amplitude on the amount of injected current suggests a contribution of active conductances. Additional support for this comes from analyzing the kinetics of the off response: its falling phase could be fitted best by a double exponential decay $\left(R^{2}=0.93\right.$ for double-exponential decay and $R^{2}=0.78$ for single-exponential decay) consisting of a fast $(\tau=2 \mathrm{~ms})$ and a slow $(\tau=40-80 \mathrm{~ms})$ component. Figure $7 \mathrm{~b}$ shows the amplitude for the two components as a function of the injected current. Whereas the slow component showed only a weak dependence on the injected current with a slight increasing trend toward hyperpolarizing currents, the amplitude of the fast component increased for current injections from +5 to $-10 \mathrm{nA}$, 
decreased for more negative currents, and became almost 0 for injections of $-30 \mathrm{nA}$. The fast component might be driven by active conductances, whereas the slow component might reflect the passive EPSP. This finding is further supported by voltage-clamp experiments (Fig. 7c). Here the membrane was clamped to $-40 \mathrm{mV}$, and the response to the light stimulus was measured. The recorded current trace showed that the light off response consists only of the slow component ( $\tau=59 \mathrm{~ms}$ ).

Ocellar input does not arrive directly at DNOVS1 but rather via a group of interneurons, called L-neurons (Strausfeld and Bassemir, 1985a). To measure the synaptic input of L-neurons to DNOVS1 directly, we recorded simultaneously from DNOVS1 and one L-neuron (Fig. 7d). As DNOVS1, the L-neuron also responds to the light on stimulus with a transient hyperpolarization followed by a hyperpolarized plateau during light stimulation and a weak transient depolarization in response to switching off the LED. The quantitative difference between the response of DNOVS1 and the simultaneously recorded L-neuron is most likely attributable to additional input from other L-neurons onto DNOVS1. It has been shown previously (Toh and Kuwabara, 1975) that the nerve projecting from the ocelli into the dorsal deuterocerebrum consists of 12 large interneurons (Lneurons) and a much higher number of thin neurons (S-neurons). Forward-fills of neurons of the ocellar nerve have suggested that at least four of the L-neurons might be presynaptic to DNOVS1 (Strausfeld and Bassemir, 1985a), but their visual response properties have not been investigated. In different insect species, it has been found that the L-neurons respond to stimulation of the ocelli with a graded and a spiking response (blowfly, Simmons et al., 1994; honeybee, Milde, 1984; Milde and Homberg, 1984). The common response of the L-neurons to light on is an inhibition and a depolarization as the light off response. The L-neuron shown here did not exhibit a pronounced light off response that could explain the amplitude of the slow component in the off response of DNOVS1. This synaptically driven depolarization is most likely mediated by other L-neurons synapsing onto DNOVS1 in addition.

The experiments described so far demonstrate that DNOVS1 receives input from two separate sources: from the photoreceptors of the compound eye via the VS cells and from photoreceptors of the ocelli via L-neurons. To test how the input signals from the two different sources (VS cells and L-neurons) are integrated, we combined these two stimuli and measured the response of DNOVS1. Figure $8 a$ shows the response to visual motion displayed in front of the compound eye, and Figure $8 b$ the response to the light on-off stimulus delivered to the ocelli. The delay between stimulus onset and response is much shorter $(12.9 \pm 1.8$ b

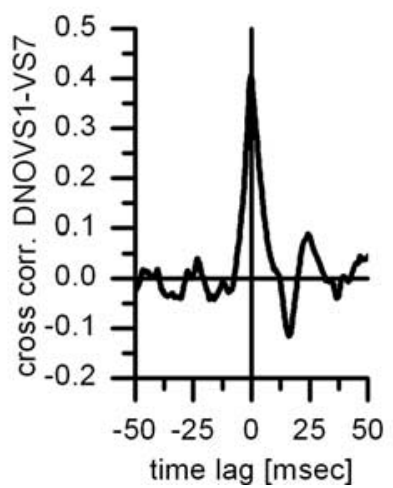

C

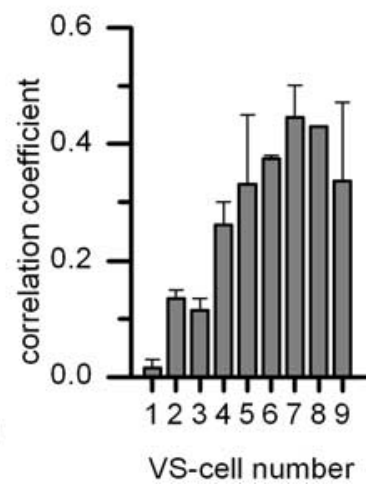

Figure 5. Cross-correlation between VS cells and DNOVS1 membrane potential. $\boldsymbol{a}$, Time course of the membrane fluctuations without any motion stimulus of DNOVS1 (black) and a simultaneously recorded VS7 (red). Note that the signals are similar. $\boldsymbol{b}$, Normalized cross-correlogram (see Materials and Methods) of the signals shown in $\boldsymbol{a}$. The peak of the cross-correlogram occurs at 作 cross-correlation follow a distribution reminiscent of the one of coupling coefficients revealed by current injection (compare with
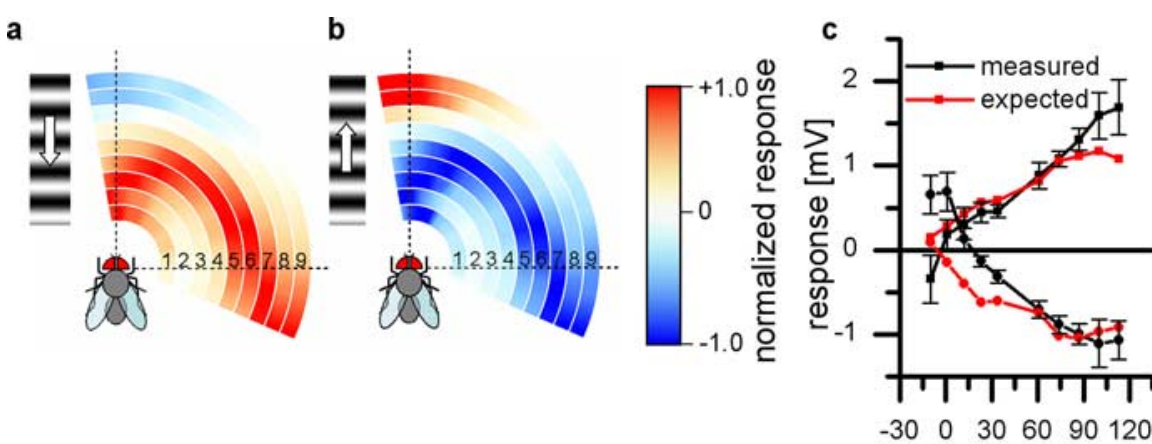

azimuth [degrees]

Figure 6. Sensitivity of VS cells to vertical grating motion as a function of the azimuth position. $\boldsymbol{a}$, Responses of VS cells to downward motion. $\boldsymbol{b}$, Responses of VS cells to upward motion. Responses were normalized with respect to the maximum (positive response maximum for downward motion and negative response maximum for upward motion) the different VS cells shifts along the azimuth according to the location of the cells dendrite in the lobula plate. VS1-VS3 had the $(n=2), \operatorname{VS2}(n=3), \operatorname{VS3}(n=8), \operatorname{VS} 4(n=7), \operatorname{VS5}(n=5), \operatorname{VS6}(n=5), \operatorname{VS7}(n=6), \mathrm{VS} 8(n=3)$, and VS9 $(n=2) . c$, Responses of DNOVS1 (black) to upward and downward motion at different azimuth positions (same data as in Fig. 1c) together with the average responses of VS1-VS9 (red) weighted by their connection strength to DNOVS1 as determined by current injection (Fig. 4).

$\mathrm{ms})$ than it is for the motion response $(42.4 \pm 1.7 \mathrm{~ms} ; n=7)$. The measured delay in the response of DNOVS1 to stimulation of the ocelli was in the range of delays reported by Parsons et al. (2006) for the response of another fly neuron called V1 after stimulation of the ocelli. This short delay indicates that the response of DNOVS1 to light pulses is mediated by a different peripheral pathway than the motion response. When we combined the two stimuli, DNOVS1 showed a response (Fig. $8 c$, black line) that is almost the linear sum of the responses to the single stimuli (Fig. $8 c$, red line). There is only a small deviation at the beginning of the stimulus in which the calculated response is stronger than the measured response.

The double recording of VS cells and DNOVS1 indicated electrical synapses between VS4-VS9 and DNOVS1. To provide additional evidence for the proposed connectivity between VS cells and DNOVS1, we injected the fluorescein-Neurobiotin dyes either into a VS cell (Fig. 9a, data from Haag and Borst, 2005) or into DNOVS1 (Fig. 9b). The labeled cell was identified under the 
a

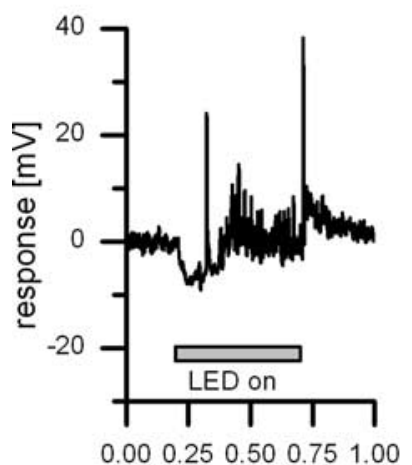

time [s]

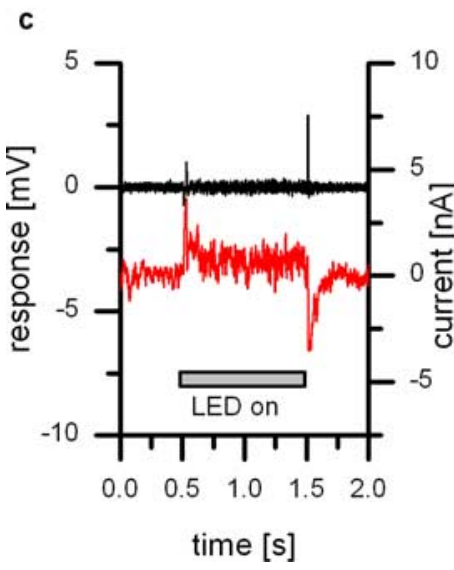

b

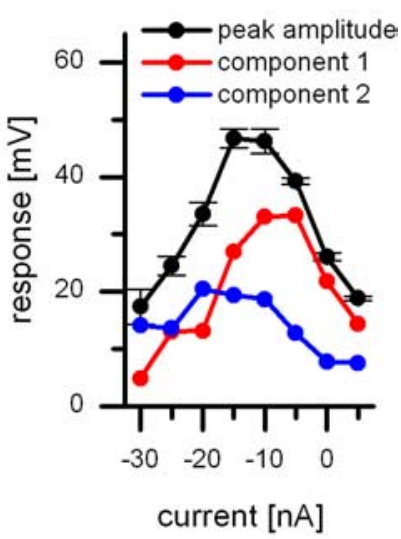

d

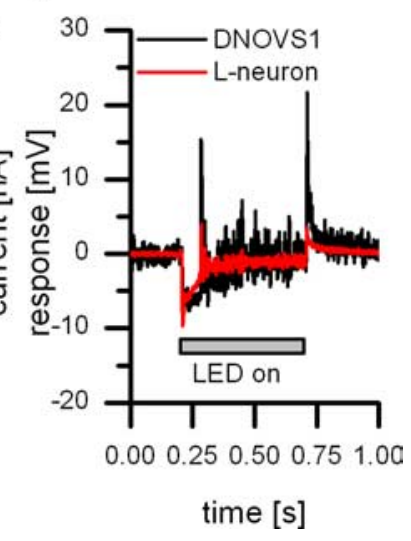

Figure 7. Responses of DNOVS1 to a stimulation of the ocelli. $\boldsymbol{a}$, Single response trace of DN0VS1. During the time indicated by the gray bar, the ocelli were stimulated by an LED. The response to light-on consists of a transient hyperpolarization followed by spike-like fluctuations of the membrane potential. Switching off the light induces a fast depolarizing peak that returns to 0 with two different time constants. $\boldsymbol{b}$, Dependence of the amplitude of the transient off response on the amount of steady-state current injected into DNOVS1 (black line). The peak amplitude showed a nearly linear dependence for current injection between -15 and $+5 \mathrm{nA}$. For more negative currents, the amplitude became reduced. Off responses could be fitted best by a double-exponential decay, with a fast ( $\tau=1.5-2.7 \mathrm{~ms}$ ) and a slow ( $\tau=40-80 \mathrm{~ms}$ ) component. The amplitude of the fast component (red line) reached the maximum for current injection of -10 to $5 \mathrm{nA}$ and became almost 0 for current injection of $-30 \mathrm{nA}$. The amplitude of the slow component (blue line) reached the maximum for injection of $-20 \mathrm{nA}$. c, Voltageclamp experiment of DNOVS1. The membrane potential (black line) was clamped to $-40 \mathrm{mV}$. The off response consists of an inward current and can be fitted with a single-exponential decay with a time constant of $62 \mathrm{~ms}$ and amplitude of $-3.7 \mathrm{nA}$. $\boldsymbol{d}$, Response of an L-neuron (red line) and response of a simultaneously recorded DNOVS1 (black line) to the stimulation of the ocelli. The L-neuron responded to light on with a fast transient hyperpolarization followed by a plateau. Switching off the LED elicited a small depolarization. Data represent the mean from five sweeps recorded in a single fly.

fluorescence microscope and a picture was taken. For the additional histological investigation, only those brains were taken in which only a single cell was visible under the fluorescence light. If the dye was injected into a VS cell (in the example shown, a VS6), the DNOVS1 was costained, and dye injection into DNOVS1 led to a costaining of VS5-VS9. These results are in accordance with an electrical coupling of VS4-VS9 and DNOVS1.

Figure $9 c-f$ show the anatomy of VS cells, DNOVS1, and an L-neuron obtained from two-photon image stacks after filling the cells with Alexa 488 and Alexa 568. In Figure 9, $c$ and $d$, three neurons are stained: a DNOVS1 (filled with Alexa 568) in red, a VS7 in green, and a VS1 in blue (both cells were filled with Alexa 488). Whereas the endings of VS7 and the dendritic branches of

DNOVS1 are in close vicinity, the endings of VS1 and DNOVS1 are in different depth $(d)$. The gap between the two cells makes it rather unlikely that there are synaptic contacts between VS1 and DNOVS1. Figure 9, $e$ and $f$, show a stained DNOVS1 (filled with Alexa 568) and an L-neuron (filled with Alexa 488). The axon terminals of the L-neuron contact the lateral dendritic branch of DNOVS1.

\section{Discussion}

The results presented above demonstrate that DNOVS1 receives synaptic input from at least two different sources, from a subset of large-field motion-sensitive VS cells as well as from the ocelli via ocellar interneurons. We also found that DNOVS1 is specifically tuned to rotational flow fields such as generated by rotation of the fly around a particular body axis. In contrast to locusts in which ocelli have been shown to play a role in visual course control of the animal (Taylor, 1981), it has been shown in the blowfly that the ocellar dorsal light response has only little influence on the visual course control (Hengstenberg, 1993; Schuppe and Hengstenberg, 1993). Using sequential stimulation of the dorsal ocelli, Parsons et al. (2006) could demonstrate in a recent study that the response of $\mathrm{V} 1$ to moving gratings is modulated by ocellar input in a rotation-specific manner. Given that V1 is a heterolateral neuron postsynaptic to VS1-VS3 that projects to the contralateral lobula plate (Kalb et al., 2006) and the neurons postsynaptic to V1 have not been identified so far, it is not clear whether these rotation-specific ocellar signals are transmitted to descending neurons, too. Because in our experiments we did not stimulate the ocelli sequentially, any discussion about the possible interplay of rotation-specific signals from the ocellar system and the compound eyes on the responses of DNOVS1 would be highly speculative. For that reason, we will concentrate the following discussion on the motion-sensitive response features of DNOVS1. In particular, we will discuss three issues raised in this context by the above findings. Can the small graded responses of DNOVS1 to motion stimuli be reliably transmitted through the cervical connective to the motor neurons in the thoracic ganglion? Can the tuning of the receptive field to rotational flow fields be explained by the described coupling to VS cells? Finally, in what respect does the representation of image motion at the level of DNOVS1 differ from the one in lobula plate cells?

\section{Small response amplitudes to motion stimuli}

We found that DNOVS1 responds to motion stimuli with a graded shift in membrane potential. However, compared with the signals of other graded potential neurons, like their presynaptic VS cells, the responses measured in the DNOVS1 were rather weak. Although it is possible to elicit action potentials by current injection (data not shown) and although the off response to ocellar stimulation showed a clear indication of active membrane conductance (Fig. 7), we did not find any evidence for a contribution of active processes to the motion response. This poses the problem of how well such small graded responses can be transmitted passively over a rather long distance of $\sim 2 \mathrm{~mm}$ along the axon into the thoracic ganglion. To address this question, instead of recording from the dendrite, we placed the electrodes in the axon close to the thoracic ganglion and recorded the response of DNOVS1 to motion stimuli. The responses of DNOVS1 recorded close to the thoracic ganglion were on average only $40 \%$ smaller than responses recorded in the dendrite $(n=2$; data not shown). Because the resting membrane potential measured in the axon was more positive than for the dendritic recording, perhaps indicating lower recording quality, the measured 
response in the axon might in fact be larger than reported here. It, thus, seems that the specific membrane conductance of the DNOVS1 axon is small enough to ensure signal transmission with little amplitude loss to its postsynaptic partner cells in the thoracic ganglion, even without amplification by voltage-dependent membrane processes.

\section{Rotational tuning and specific connectivity to VS cells}

Our statements about the connectivity of DNOVS1 to the VS cells are based on two findings: first we measured the potential response to current injection of both polarities and in both directions (Fig. 4), and next we measured the correlation strength of the signals in both cells without stimulation (Fig. 5). Both types of experiments revealed that DNOVS1 is substantially connected to VS4-VS9, with maximum coupling to VS7 and VS8 and only residual coupling to the frontal cells VS1-VS3. The measured weak coupling between VS1-VS3 and DNOVS1 might be attributable to the coupling of the VS cells to each other (Haag and Borst, 2004). Current injection into VS3 leads to a response in VS4, which is then transmitted to DNOVS1, and consequently would not indicate a direct coupling between VS3 and DNOVS1.

The measured sensitivity of DNOVS1 to vertical moving stimuli matches quite well the receptive field organization of VS7 (Krapp et al., 1998). Nevertheless, the coupling coefficients determined by current injection show that DNOVS1 receives not only input from VS7 onto DNOVS1 but also from VS4 to VS9 with different coupling strength. To see whether the coupling coefficients determined this way are in accordance with the azimuthal sensitivity distribution of DNOVS1 (Fig. 1), we determined the corresponding sensitivity profile of all VS cells except VS10 using the exact same stimulus device (Fig. 6). To avoid any ambiguities in identifying VS cells, we also took precise measurements of the location of the ventral dendrite along the mediolateral axis within the lobula plate (Farrow, 2005). Summing up the sensitivities of the VS cells weighted according to the coupling strengths led to a linear prediction of the azimuth sensitivity of DNOVS1 that is shown next to the measured one in Figure 6. Both profiles can be seen to match quite well, with a peak sensitivity for lateral positions decreasing toward frontal positions. If DNOVS1 were to integrate over all VS cells with equal strength, a rather homogeneous sensitivity for downward motion at all azimuth positions would result (data not shown). In the frontal part, however, a significant deviation of the measured DNOVS1 sensitivity from the expected one can be observed: whereas the measured sensitivity exhibits a 0 crossing at $\sim 15^{\circ}$ and inverts its sign at frontal positions, the expected sensitivity for upward motion is $\sim 0$ at frontal positions. This means that, from the connectivity pattern, DNOVS1 is expected to respond to vertical motion at lateral positions regardless of motion displayed in front of the fly. The neuron would, thus, not discriminate between a translational flow field originating by an upward motion of the fly ("lift") and a rotational flow field originating by rotation of the fly around its longitudinal axis ("roll"). Quite in contrast, it responds most strongly to lateral downward motion and frontal upward motion, resulting in a specific tuning to rotational flow fields, in which the pole of the rotation is located at $15^{\circ}$ azimuth (Fig. 2). Mechanis- tically, this might be explained by reciprocal inhibitory connections between the most frontal and most lateral VS cells postulated in a recent study on current injection experiments from pairs of VS cells (Haag and Borst, 2004). Such an inhibitory loop can explain the slight preference for upward motion of lateral VS cells in frontal positions of the visual field. If such connections are located at the axon terminal regions of VS cells, the effect of this reciprocal inhibition and the resulting sign inversion would be underestimated in recordings from mid-axonal positions of the cells but would show up much more pronounced in the dendrite of a postsynaptic cell such as DNOVS1. Another possibility to explain the above phenomenon is the existence of chemical synapses between VS cells and DNOVS1: whereas our experiments have revealed no positive evidence for such connections, chemical synapses have been anatomically described by Strausfeld and Bassemir (1985a). Although nothing is known about the strength and nature of these synapses, their effects might combine with the electrical coupling and become visible during visual motion stimulation, leading to a rotational tuning of DNOVS1 as found in this study. A third possibility that cannot be excluded at present is the connection of DNOVS1 to other neurons of the fly optic lobes that have escaped our notice so far. A final decision between the above alternatives will have to wait until a complete description of the circuitry will be available from serial block face scanning electron microscopy technology (Denk and Horstmann, 2004).

\section{Functional significance of rotational tuning}

Aside from the problem discussed above of how to explain the observed receptive field properties of DNOVS1 given the observed connectivity to VS cells, another interesting question is how the representation of image flow at the level of DNOVS1 differs from the one in the presynaptic set of VS cells. Although our stimulation device did not allow displaying rotational stimuli, we approximated such stimuli by showing vertical image motion going upward and downward at different positions simultaneously and compared the responses of DNOVS1 to combined stimuli with the sum of responses to the individual stimulus components. As was observed with ocellar stimulation, the outcome of these experiments revealed a perfectly linear response behavior of DNOVS1 (Fig. 2). Although such a linear superposition of individual response components might not sound as an indication for a high degree of specificity, it is indeed much different 

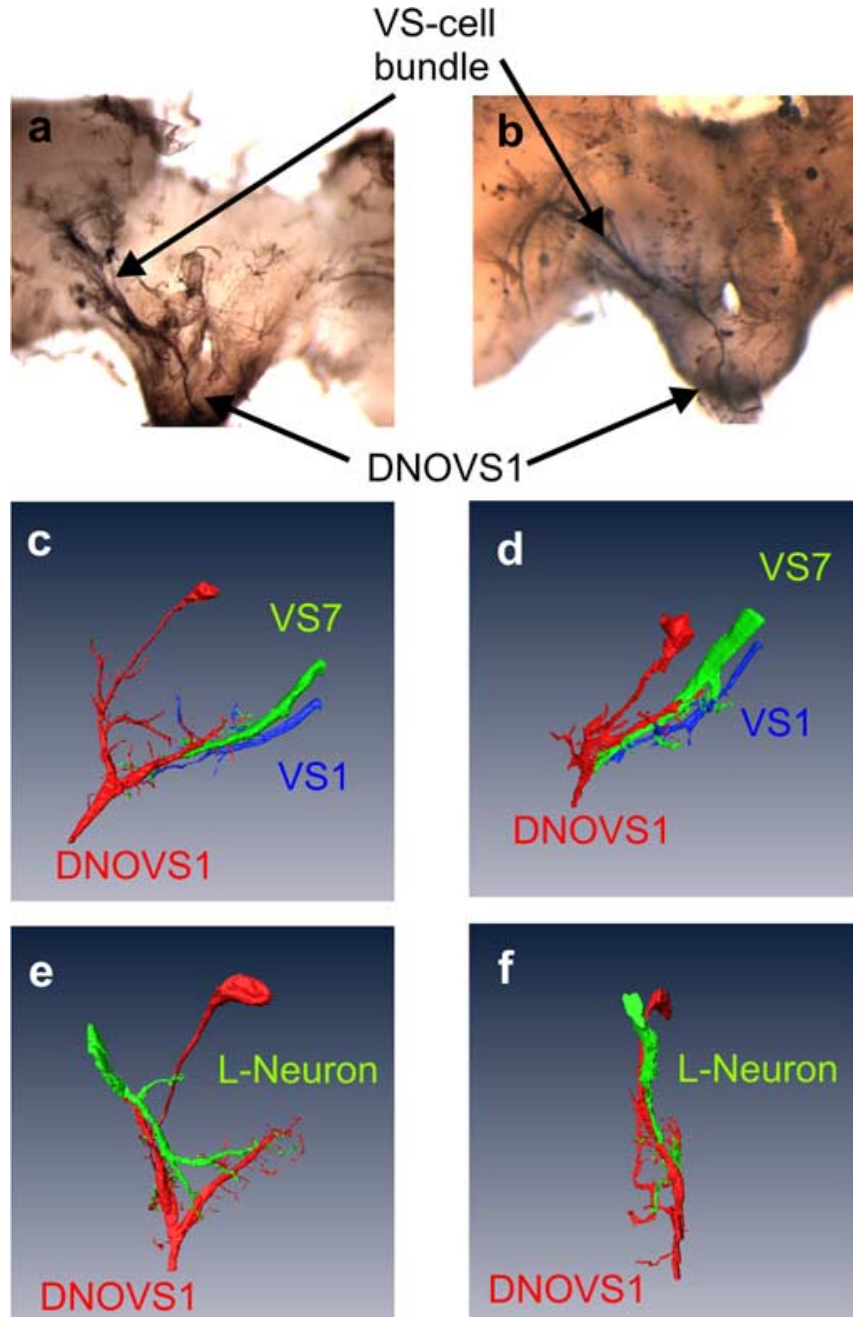

Figure 9. Anatomy and dye coupling. $\boldsymbol{a}$, Neurobiotin staining of a VS6. Besides the costaining of adjacent VS cells, DNOVS1 was found to be labeled, too (data from Haag and Borst, 2005). $\boldsymbol{b}$, Injection of Neurobiotin into DNOVS1 led to a specific costaining of VS cells. VS6 and VS7 showed the strongest staining, and VS5, VS8, and VS9 were stained weakly. This confirms the results about the coupling strength between DNOVS1 and the VS cells (see Fig. 2c). c, Twophoton imaging of DNOVS1 and VS cells. DNOVS1 (red) was filled with the red fluorescent dye Alexa 568, and VS1 (blue) and VS7 (green) were filled with the green fluorescent Alexa 488. Shown is a $x-y$ projection. The side view $(\boldsymbol{d})$ shows that the axon terminal of VS7 and the dendrite are in close vicinity. $\boldsymbol{e}, x-y$ projection of DNOVS1 (red) and an L-neuron (green). $\boldsymbol{f}$, Same as $\boldsymbol{e}$ but in a side view. Two-photon image stacks are subject to iso-surface rendering by Amira software package (see Materials and Methods)

from the strongly sublinear response summation observed in the vertical cells of the lobula plate. There, the responses of VS cells tend to saturate with increasing size of stimulated area (Haag et al., 1992). Furthermore, stimulation of V1 revealed that, unlike DNOVS1, V1 responds almost as strongly to large-field downward motion as to rotational flow fields that perfectly match its receptive field (Karmeier et al., 2003). Thus, a linear response summation in DNOVS1 increases its response selectivity compared with the one of lobula plate cells substantially. A quantitative analysis of this effect requires stimulation of all VS cells with rotational flow fields as well as with translational ones and comparing the resulting flow-field selectivity with the one observed at the level of descending neurons such as DNOVS1. Such an analysis will further elucidate how the neural representation of flow fields changes at the various processing levels from lobula plate through descending down to motor neurons in the neck and thorax of the fly (for first results on flow fields of neck motoneurons, see Huston and Krapp, 2003; Krapp and Huston, 2005).

\section{References}

Borst A, Haag J (1996) The intrinsic electrophysiological characteristics of fly lobula plate tangential cells. I. Passive membrane properties. J Comput Neurosci 3:313-336.

Borst A, Haag J (2002) Neural networks in the cockpit of the fly. J Comp Physiol 188:419-437.

Chan WP, Prete F, Dickinson MH (1998) Visual input to the efferent control system of a fly's "gyroscope." Science 280:289-292.

Denk W, Horstmann H (2004) Serial block-face scanning electron microscopy to reconstruct three-dimensional tissue nanostructure. PLoS Biol 2:1900-1909.

Denk W, Strickler JH, Webb WW (1990) Two-photon laser scanning fluorescence microscopy. Science 248:73-76.

Eckert H, Dvorak DR (1983) The centrifugal horizontal cells in the lobula plate of the blowfly Phaenicia sericata. J Insect Physiol 29:547-560.

Farrow K (2005) Lateral interactions and receptive field structure of lobula plate tangential cells in the blowfly. $\mathrm{PhD}$ thesis, Ludwig-MaximiliansUniversität

Farrow K, Haag J, Borst A (2003) Input organization of multifunctional motion sensitive neurons in the blowfly. J Neurosci 23:9805-9811.

Farrow K, Borst A, Haag J (2005) Sharing receptive fields with your neighbors: tuning the vertical system cells to wide field motion. J Neurosci 25:3985-3993.

Farrow K, Haag J, Borst A (2006) Nonlinear, binocular interactions underlying flow field selectivity of a motion-sensitive neuron. Nat Neurosci 9:1312-1320.

Franz MO, Krapp HG (2000) Wide-field, motion-sensitive neurons and matched filters for optic flow fields. Biol Cybern 83:185-197.

Gilbert C, Gronenberg W, Strausfeld NJ (1995) Oculomotor control in calliphorid flies: head movements during activation and inhibition of neck motor neurons corroborate neuroanatomical predictions. J Comp Neurol 361:285-297.

Gronenberg W, Strausfeld NJ (1990) Descending neurons supplying the neck and flight motor of diptera: physiological and anatomical characteristics. J Comp Neurol 302:973-991.

Gronenberg W, Milde JJ, Strausfeld NJ (1995) Oculomotor control in calliphorid flies: organization of descending neurons to neck motor neurons responding to visual stimuli. J Comp Neurol 361:267-284.

Haag J (1994) Aktive und passive Membraneigenschaften bewegungsempfindlicher Interneurone der Schmeissfliege Calliphora erythrocephala. $\mathrm{PhD}$ thesis, Eberhard-Karls-Universität zu Tübingen.

Haag J, Borst A (2001) Recurrent network interactions underlying flowfield selectivity of visual interneurons. J Neurosci 21:5685-5692.

Haag J, Borst A (2002) Dendro-dendritic interactions between motionsensitive large-field neurons in the fly. J Neurosci 22:3227-3233.

Haag J, Borst A (2003) Orientation tuning of motion-sensitive neurons shaped by vertical-horizontal network interactions. J Comp Physiol 189:363-370.

Haag J, Borst A (2004) Neural mechanism underlying complex receptive field properties of motion-sensitive interneurons. Nat Neurosci $7: 628-634$.

Haag J, Borst A (2005) Dye-coupling visualizes networks of large-field motion-sensitive neurons in the fly. J Comp Physiol 191:445-454.

Haag J, Egelhaaf M, Borst A (1992) Dendritic integration of motion information in visual interneurons of the blowfly. Neurosci Lett 140:173-176.

Haag J, Vermeulen A, Borst A (1999) The intrinsic electrophysiological characteristics of fly lobula plate tangential cells. III. Visual response properties. J Comput Neurosci 7:213-234.

Haag J, Denk W, Borst A (2004) Fly motion vision is based on Reichardt detectors regardless of the signal-to-noise ratio. Proc Natl Acad Sci USA 101:16333-16338.

Hausen K (1977) Struktur, Funktion und Konnektivität bewegungsempfindlicher Interneurone im dritten optischen Neuropil der Schmeissfliege Calliphora erythrocephala. PhD thesis, Eberhard-Karls-Universität zu Tübingen.

Hausen K (1981) Monocular and binocular computation of motion in the lobula plate of the fly. Verh Dtsch Zool Ges 74:49-70.

Hausen K (1982) Motion sensitive interneurons in the optomotor system of 
the fly. I. The horizontal cells: structure and signals. Biol Cybern 45:143-156.

Hausen K (1984) The lobula-complex of the fly: structure, function and significance in visual behaviour. In: Photoreception and vision in invertebrates (Ali MA, ed). pp 523-559. New York: Plenum.

Hengstenberg R (1977) Spike response of "non-spiking" visual interneurone. Nature 270:338-340.

Hengstenberg R (1993) Multisensory control in insect oculomotor systems. Rev Oculomot Res 5:285-298.

Hengstenberg R, Hausen K, Hengstenberg B (1982) The number and structure of giant vertical cells (VS) in the lobula plate of the blowfly Calliphora erytrocephala. J Comp Physiol 149:163-177.

Horstmann W, Egelhaaf M, Warzecha AK (2000) Synaptic interactions increase optic flow specificity. Eur J Neurosci 12:2157-2165.

Huston SJ, Krapp HG (2003) Visual receptive field of a fly neck motorneuron. In: Goettingen neurobiology report 2003 (Elsner N, Schnitzler HU, eds), p 559. Stuttgart, Germany: Thieme.

Kalb J, Egelhaaf M, Kurtz R (2006) Robust integration of motion information in the fly visual system revealed by single cell photoablation. J Neurosci 26:7898-7906.

Karmeier K, Krapp HG, Egelhaaf M (2003) Robustness of the tuning of fly visual interneurons to rotatory optic flow. J Neurophysiol 90:1626-1634.

Kimpo RR, Theunissen FE, Doupe AJ (2003) Propagation of correlated activity through multiple stages of a neural circuit. J Neurosci 23:5750-5761.

Krapp HG, Hengstenberg R (1996) Estimation of self-motion by optic flow processing in single visual interneurons. Nature 384:463-466.

Krapp HG, Huston SJ (2005) Encoding self-motion: from visual receptive fields to motor neuron response maps. Presented at 30th Goettingen Neurobiology Conference, Sixth Meeting of the German Neuroscience Society, Goettingen, Germany, February.

Krapp HG, Hengstenberg B, Hengstenberg R (1998) Dendritic structure and receptive-field organization of optic flow processing interneurons in the fly. J Neurophysiol 79:1902-1917.

Milde JJ (1984) Ocellar interneurons in the honeybee structure and signals of L-neurons. J Comp Physiol 154:683-693.
Milde JJ, Homberg U (1984) Ocellar interneurons in the honeybee: characteristics of spiking L-neurons. J Comp Physiol 155:151-160.

Milde JJ, Strausfeld NJ (1986) Visuo-motor pathways in arthropods. Naturwissenschaften 73:151-154.

Parsons MM, Krapp HG, Laughlin SB (2006) A motion-sensitive neurone responds to signals from the two visual systems of the blowfly, the compound eyes and ocelli. J Exp Biol 209:4464-4474.

Pierantoni R (1976) A look into the cock-pit of the fly. Cell Tissue Res 171:101-122.

Schuppe H, Hengstenberg R (1993) Optical properties of the ocelli of Calliphora erythrocephala and their role in the dorsal light response. J Comp Physiol 173:143-149.

Simmons PJ, Jian S, Rind FC (1994) Characterisation of large second-order ocellar neurons of the blowfly Calliphora erythrocephala. J Exp Biol 191:231-245.

Strausfeld NJ, Bassemir UK (1985a) Lobula plate and ocellar interneurons converge onto a cluster of descending neurons leading to neck and leg motor neuropil in Calliphora erythrocephala. Cell Tissue Res 240:617-640.

Strausfeld NJ, Bassemir UK (1985b) The organization of giant horizontalmotion-sensitive neurons and their synaptic relationships in the lateral deutocerebrum of Calliphora erythrocephala and Musca domestica. Cell Tissue Res 242:531-550.

Strausfeld NJ, Gronenberg W (1990) Descending neurons supplying the neck and flight motor of diptera: organization and neuroanatomical relationships with visual pathways. J Comp Neurol 302:954-972.

Strausfeld NJ, Seyan HS (1985) Convergence of visual, haltere, and prosternal inputs at neck motor neurons of Calliphora erythrocephala. Cell Tissue Res 240:601-615.

Strausfeld NJ, Seyan HS, Milde JJ (1987) The neck motor system of the fly Calliphora erythrocephala. J Comp Physiol 160:205-224.

Taylor CP (1981) Contribution of compound eyes and ocelli to steering of locusts in flight. II. Timing changes in flight motor units. J Exp Biol 93:19-31.

Toh Y, Kuwabara M (1975) Synaptic organization of the fleshfly ocellus. J Neurocytol 4:271-287. 